\title{
Influência das propriedades físico-químicas dos iogurtes no desenvolvimento da erosão dental
}

\author{
Influence of the physical-chemical properties of yogurts \\ on the development of dental erosion \\ Influencia de las propiedades fisicoquímicas de los yogures \\ en el desarrollo de la erosión dental
}

\author{
Camila Lima de OLIVEIRA ${ }^{\mathbf{1}}$ \\ Flávio de Almeida ANDRADE ${ }^{1}$ \\ José de Alencar FERNANDES NETO ${ }^{2}$ \\ Michelangela Suelleny de Caldas NOBRE ${ }^{3}$ \\ Tiago Almeida de OLIVEIRA ${ }^{4}$ \\ Maria Helena Chaves de Vasconcelos CATÃO ${ }^{5}$ \\ ${ }^{1}$ Graduando(a) em Odontologia, Departamento de Odontologia, \\ Universidade Estadual da Paraíba - UEPB, 58429-500, Campina Grande-PB, Brasil \\ ${ }^{2}$ Mestrando do Programa de Pós-Graduação em Odontologia, Departamento de Odontologia, \\ Universidade Estadual da Paraíba - UEPB, 58429-500 Campina Grande-PB, Brasil \\ ${ }^{3}$ Professora Mestre em Ciências Farmacêuticas, Departamento de Medicina, \\ Faculdade Integrada de Patos - FIP, 58.704-000 Patos-PB, Brasil \\ Técnica de Química, Departamento de Química, \\ Universidade Estadual da Paraíba - UEPB, 58429-500 Campina Grande - PB, Brasil \\ ${ }^{4}$ Professor Doutor em Ciências, Linha De Concentração Estatística e Experimentação Agronômica, \\ Departamento de Estatística, Universidade Estadual da Paraíba - UEPB, 58429-500 Campina Grande-PB, Brasil \\ ${ }^{5}$ Professora Doutora do Programa de Pós-Graduação em Odontologia, \\ Universidade Estadual da Paraíba - UEPB, 58429-500 Campina Grande-PB, Brasil
}

\section{Resumo}

Introdução: A ingestão de bebidas ácidas está relacionada à erosão dentária, perda crônica de tecidos duros dentários. Objetivo: Mensurar o pH, a acidez total titulável (ATT), o ácido lático (AL) e a quantidade de sólidos solúveis totais (SST) de iogurtes industrializados, em duas temperaturas diferentes. Material e método: Foram obtidos vinte iogurtes, de diferentes marcas comerciais e sabores. Metade das amostras foram mantidas refrigeradas $\left( \pm 9^{\circ} \mathrm{C}\right)$, enquanto as demais, colocadas em temperatura ambiente $\left( \pm 27^{\circ} \mathrm{C}\right)$, por três horas. A mensuração do $\mathrm{pH}$ endógeno foi realizada por meio do ph-metro digital, a acidez total titulável e o ácido lático analisados por titulação e a leitura dos sólidos solúveis totais feita pelo refratômetro de Abbe. Os testes foram realizados em triplicata. Resultados: Todos os iogurtes apresentaram $\mathrm{pH}$ inferior ao valor crítico para a dissolução do esmalte dentário $(5,5)$. As médias máxima e mínima obtidas para a acidez total titulável e o ácido lático foram respectivamente $(13,74$ e 6,34$)$ e $(1,22$ e 0,57), variando o SST entre 9,70 e 20,20. Houve variação estatística significativa nos valores do pH, da acidez total titulável, do ácido lático e dos sólidos solúveis totais, quando iogurtes foram submetidos à diferentes temperaturas. Conclusão: Os resultados sugerem que se consumidos com frequência, os iogurtes podem contribuir para o desenvolvimento da erosão dental.

Descritores: Concentração de Íons de Hidrogênio; Acidez; Dieta; Erosão Dentária.

\begin{abstract}
Introduction: Ingestion of acidic beverages is related to dental erosion, chronic loss of hard dental tissues. Objective: To measure $\mathrm{pH}$, total titratable acidity, lactic acid and the amount of total soluble solids of industrialized yoghurts, at two different temperatures. Material and method: Twenty yoghurts of different brands and flavors were obtained. Half of the samples were kept refrigerated $\left( \pm 9^{\circ} \mathrm{C}\right)$, while the others were placed at room temperature $\left( \pm 27^{\circ} \mathrm{C}\right)$ for three hours. The endogenous pH was measured using the digital $\mathrm{pH}$ meter, titratable total acidity and lactic acid analyzed by titration and the reading of the total soluble solids by the Abbe refractometer. The tests were performed in triplicate. Results: All the yoghurts presented pH lower than the critical value for the dissolution of the dental enamel $(5,5)$. The maximum and minimum averages obtained for titratable total acidity and lactic acid were respectively (13.74 and 6.34) and (1.22 and 0.57), with the total soluble solids varying between 9.70 and 20.20. There was a statistically significant variation in $\mathrm{pH}$ values, total titratable acidity, lactic acid and total soluble solids, when yoghurts were submitted to different temperatures. Conclusion: The results suggest that if consumed frequently, yogurts may contribute to the development of dental erosion.
\end{abstract}

Descriptors: Hydrogen-Ion Concentration; Acidity; Diet; Tooth Erosion.

\section{Resumen}

Introducción: El consumo de bebidas ácidas está relacionado con la erosión dental, pérdida crónica de los tejidos duros de los dientes. Objetivo: medir pH, la acidez titulable, ácido láctico y la cantidad de sólidos solubles de yogures industrializados, a dos temperaturas diferentes. Material y método: Se obtuvieron veinte yogures, de diferentes marcas y sabores. La mitad de las muestras se mantuvieron refrigeradas $\left( \pm 9{ }^{\circ} \mathrm{C}\right)$ mientras que la otra, colocada a temperatura ambiente $\left( \pm 27^{\circ} \mathrm{C}\right)$ durante tres horas. La medición de pH endógeno se realizó mediante el pH-metro digital, la acidez titulable analizó por titulación y la lectura de el cantidad de sólidos solubles por refractómetro de Abbe. Los ensayos se realizaron por triplicado. Resultados: Todos los yogures tenían un $\mathrm{pH}$ por debajo del valor crítico para la disolución del esmalte de los dientes (5.5). Los promedios máximos y mínimos de la acidez titulable y ácido láctico fueron respectivamente (13.74 y 6.34) y (1.22 y 0.57), variando la SST entre 9.70 y 20.20. Hubo variación estadísticamente significativa en los valores de pH, la acidez titulable, ácido láctico y los sólidos solubles totales cuando yogures fueron sometidos a diferentes temperaturas. Conclusión: Los resultados sugieren que los yogures pueden contribuir al desarrollo de la erosión dental, cuando son consumidos con frecuencia.

Descriptores: Concentración de Iones de Hidrógeno; Acidez; Dieta; Erosión de los Dientes.

\section{INTRODUÇÃO}

No cenário mundial foram verificadas mudanças nos hábitos alimentares dos indivíduos. A ingestão de bebidas industrializadas repercute negativamente na saúde bucal dos indivíduos, contribuindo para o surgimento de lesões orais cariosas e não cariosas, que podem ocasionar comprometimento da função e da estética ${ }^{1}$. 
Diversas bebidas ácidas estão correlacionadas ao processo de erosão dental ${ }^{2}$. Tais produtos, são constituídos de uma diversidade de ácidos, substâncias de $\mathrm{pH}$ baixo (inferior a 5) que favorece o processo de desgaste dentário ${ }^{3}$. Os fatores etiológicos extrínsecos da erosão dental envolvem remédios ácidos como a vitamina $\mathrm{C}$, além do consumo de comidas e bebidas ácidas. As causas intrínsecas estão relacionadas à anorexia nervosa e bulimia, bem como a qualquer desordem gastroesofágica ${ }^{4}$.

Perda de brilho e superfície lisa em forma de "U" ou pires são características da erosão dental ${ }^{5}$, problema clínico significativo, que pode ser encontrado em indivíduos de todas as faixas etárias ${ }^{6}$. Por ser considerada uma doença multifatorial (resultante da interação de fatores químicos, biológicos e comportamentais), estudos tem demonstrado diferença na prevalência da patologia entre adolescentes e adultos jovens ${ }^{7}$.

A bebida láctea consiste em um produto constituído de leite e/ ou derivados de leite, podendo ser adicionados à composição ingredientes como iogurtes, soro, aromatizantes, corantes, edulcorantes e polpa de fruta. A possibilidade de adição de leite em pó, proteínas, vitaminas e sais minerais enriquece nutricionalmente os iogurtes, no entanto, a presença de ácido em sua composição pode contribuir para desmineralização do elemento dentário ${ }^{8,9}$.

A base científica utilizada para fundamentar o estudo permite acreditar que o consumo de bebidas ácidas favorece o desenvolvimento da erosão dental. Diante do exposto, o estudo buscou avaliar o $\mathrm{pH}$, a acidez total titulável (ATT), a quantidade de ácido lático (AL) e os sólidos solúveis totais (SST) de iogurtes, contribuindo para ampliação do número de documentos que analisam as propriedades físico-químicas de bebidas ácidas e sua correlação com a erosão dental, a fim de evidenciar aos cirurgiões-dentistas a importância da orientação dietética dos pacientes na prevenção de patologias orais.

\section{MATERIAL E MÉTODO}

O presente estudo é do tipo experimental in vitro e fez uso da técnica da observação direta em laboratório.

Em pontos comerciais situados na cidade de Campina Grande-PB, no período de maio de 2016, foram obtidos 20 iogurtes, de diferentes marcas comerciais e sabores (Quadro 1).

Quadro 1. Distribuição dos iogurtes de acordo com as marcas comerciais e sabores

\begin{tabular}{|c|c|}
\hline MARCAS COMERCIAIS & SABORES \\
\hline \multirow[b]{2}{*}{ A- CARIRI ${ }^{\circledR}$} & (A1.1) Morango \\
\hline & (A1.2) Mel \\
\hline \multirow[b]{2}{*}{ B- LEBON ${ }^{\circledR}$} & (B 1.1) Morango \\
\hline & (B 1.2) Ameixa \\
\hline \multirow{2}{*}{ C- NINHO ${ }^{\circledR}$} & (C 1.1) Morango \\
\hline & (C1.2) Maçã e banana \\
\hline \multirow[b]{2}{*}{ D- ÍSIS ${ }^{\circledR}$} & (D1.1) Morango \\
\hline & (D1.2) Ameixa \\
\hline \multirow[b]{2}{*}{ E- DANONE ${ }^{\circledR}$} & (E 1.1) Morango \\
\hline & (E1.2) Vitamina de frutas \\
\hline
\end{tabular}

A pesquisa foi realizada no Laboratório de Química da Universidade Estadual da Paraíba. Os experimentos foram realizados inicialmente nas amostras mantidas refrigeradas $\left( \pm 9^{\circ} \mathrm{C}\right)$ (10 iogurtes), seguido das que foram expostas à temperatura ambiente $\left( \pm 27^{\circ} \mathrm{C}\right)(10$ iogurtes $)$, por três horas. Todos os testes foram realizados em triplicata.

A mensuração do $\mathrm{pH}$ endógeno ocorreu por meio $\mathrm{pH}$ metro-digital (Modelo TEC-5, 110/220V, 7W - TECNAL® Equipamentos), aparelho calibrado de acordo com as orientações do fabricante. Após abertura da embalagem, foi transferido $50 \mathrm{ml}$ de iogurte para um becker e procedendo-se às medições. Através da imersão do eletrodo foi obtido o valor do $\mathrm{pH}$, registrado em ficha clínica.

Para a determinação da ATT e do AL, utilizou-se a metodologia adotada pelo Instituto Adolfo Lutz. Foram preparados soluções de Fenolftaleína $1 \%$ e Hidróxido de sódio $0,1 \mathrm{M}$ de $\mathrm{NaOH}$, correspondendo a 0,9635 o valor do fator de correção, obtido após a etapa da padronização dos reagentes. Com a pesagem de $5 \mathrm{~g}$ do iogurte e posterior adição de $50 \mathrm{~mL}$ de água destilada, adicionou-se a amostra 4 gotas fenolftaleína (homogeneizando até a dissolução, por meio de um bastão de vidro). Encheu-se a bureta de solução de $0,1 \mathrm{M}$ de $\mathrm{NaOH}$ e por meio da adição de gotas da solução de hidróxido de sódio à solução realizou-se a titulação, cujo gotejamento ocorreu até a obtenção da coloração rósea.

Os cálculos da ATT bem como do AL foram obtidos por meio de fórmula específica, que expressaram em porcentagem o valor das respectivas variáveis.

Para a obtenção da quantidade de sólidos solúveis totais, utilizou-se o refratômetro de Abbe (Brix/RI- Chek Refractometer - Reichert®, Japan), aparelho calibrado à temperatura ambiente, com água destilada. $\mathrm{O}$ refratômetro forneceu a leitura do grau Brix, expressando em porcentagem a quantidade de sólidos solúveis totais contidos na amostra.

As observações foram expressas em médias e desvios padrão, sendo submetidos à análise via modelos lineares clássicos (ML) com uso de análise de variância (ANOVA), para as variáveis SST, pH, ATT e modelos lineares generalizados (MLG) com uso de análise de deviance (ANODEV) sob distribuição Gama, para AL. Os resíduos foram analisados e submetidos aos testes de Shapiro-Wilk para verificar normalidade e Levene para checar homocedasticidade. A adequabilidade do modelo linear generalizado aplicado as variáveis foi realizada via gráfico half normal com envelope simulado. A significância utilizada para todas as análises foi de $5 \%(\mathrm{p}<0,05)$ com $95 \%$ de grau de confiança. O teste Tukey foi aplicado para a média de todas as variáveis ao nível de 5\% de significância.

\section{RESULTADOS}

No que se refere ao $\mathrm{AL}$, os valores variaram entre 0,57 e 1,22. Nos iogurtes A1.1, D1.1 e D1.2 foram verificadas diferenças estatísticas significativas nos valores do AL quando os iogurtes foram submetidos a temperaturas distintas. Nas referidas situações, os iogurtes mantidos à $\left( \pm 27^{\circ} \mathrm{C}\right)$ apresentaram maior valor de AL. No caso do B 1.1 e B 1.2 (iogurtes de mesma marca comercial, com sabores diferentes), foram verificados diferenças estatísticas significativa no valor do AL tanto a $\left( \pm 9^{\circ} \mathrm{C}\right)$ quanto a $\left( \pm 27^{\circ} \mathrm{C}\right)$ (Tabela 1$)$.

Para o $\mathrm{pH}$, o valor máximo e mínimo encontrados foram respectivamente 4,75 e 4,06 , de modo que os iogurtes refrigerados apresentaram $\mathrm{pH}$ superior aos que foram mantidos em temperatura ambiente. Foi possível constatar também a influência do sabor das bebidas nos valores encontrados. Tanto a $\left( \pm 9^{\circ} \mathrm{C}\right)$ quanto a $\left( \pm 27^{\circ} \mathrm{C}\right)$ foram verificadas diferenças estatísticas significativas no $\mathrm{pH}$ de iogurtes de mesma marca comercial com sabores diferentes (Tabela 1).

$\mathrm{Na}$ ATT a interferência da temperatura foi significativa no caso dos iogurtes B 1.1, C1.1, D 1.1 e D 1.2, onde a acidez foi maior quando as bebidas foram expostas à $\left( \pm 27^{\circ} \mathrm{C}\right)$. Para os iogurtes de mesma marca comercial com sabores diferentes, a diferença no valor da acidez foi significativa no caso dos iogurtes $\mathrm{B}$ (tanto a $\left( \pm 9^{\circ} \mathrm{C}\right)$ quanto a 
$\left( \pm 27^{\circ} \mathrm{C}\right)$ e nos iogurtes $\mathrm{C}$ (quando expostos à $\left.\left( \pm 27^{\circ} \mathrm{C}\right)\right)$. Os valores da ATT variaram entre 6,34 e 13,74 (Tabela 1).

Tabela 1. Valores médios e desvio padrão do AL, pH, ATT, e SST de iogurtes, submetidos a temperaturas distintas $\left( \pm 9^{\circ} \mathrm{C}\right)$ e $\left( \pm 27^{\circ} \mathrm{C}\right)$

\begin{tabular}{|c|c|c|c|c|c|c|c|c|}
\hline \multirow{4}{*}{$\begin{array}{l}\text { Ensaios } \\
\text { (Valores } \\
\text { Médios) }\end{array}$} & \multicolumn{8}{|c|}{ Parâmetros } \\
\hline & \multicolumn{2}{|c|}{ AL $\left(M^{\prime} G^{*}\right)$} & \multicolumn{2}{|c|}{$\mathrm{pH}\left(\mathrm{ML}^{\star \star *}\right)$} & \multicolumn{2}{|c|}{ ATT $\left(M L^{\star \star}\right)$} & \multicolumn{2}{|c|}{ SST $\left(M L^{\star \star}\right)$} \\
\hline & & & & Tempe & aturas $\left({ }^{\circ} \mathrm{C}\right)$ & & & \\
\hline & $\begin{array}{c}9 \\
\text { (D.P) }\end{array}$ & $\begin{array}{l}27 \\
\text { (D.P) }\end{array}$ & $\begin{array}{c}9 \\
\text { (D.P) }\end{array}$ & $\begin{array}{c}27 \\
\text { (D.P) }\end{array}$ & $\begin{array}{c}9 \\
\text { (D.P) }\end{array}$ & $\begin{array}{l}27 \\
\text { (D.P) }\end{array}$ & $\begin{array}{c}9 \\
\text { (D.P) }\end{array}$ & $\begin{array}{c}27 \\
\text { (D.P) }\end{array}$ \\
\hline \multirow{2}{*}{ A1.1 } & $0,66 \mathrm{deB}$ & $0,71 \mathrm{dA}$ & $4,68 \mathrm{bA}$ & $4,07 \mathrm{fB}$ & $7,32 \mathrm{dA}$ & $7,95 \mathrm{dA}$ & $20,20 \mathrm{aA}$ & $13,56 \mathrm{~dB}$ \\
\hline & $(0,0308)$ & $(0,0286)$ & $(0,0003)$ & $(0,0003)$ & $(0,0028)$ & $(0,0026)$ & $(0,0009)$ & $(0,0014)$ \\
\hline \multirow{2}{*}{ A1.2 } & 0,65 deA & $0,67 \mathrm{deA}$ & $4,31 \mathrm{eA}$ & $4,09 \mathrm{eB}$ & $7,18 \mathrm{deA}$ & $7,49 \mathrm{dA}$ & $18,36 \mathrm{abA}$ & $19,40 \mathrm{aA}$ \\
\hline & $(0,0314)$ & $(0,03 \mathrm{C}$ & & $(0,0003)$ & $(0,0028)$ & $(0,0027)$ & $(0,0010)$ & $(0,0010)$ \\
\hline \multirow{2}{*}{ B1.1 } & $1,20 \mathrm{aA}$ & $1,22 \mathrm{aA}$ & $4,68 \mathrm{bA}$ & $4,06 \mathrm{fB}$ & $13,41 \mathrm{aB}$ & $13,74 a A$ & $13,56 \mathrm{dA}$ & $13,40 \mathrm{dA}$ \\
\hline & $(0,0169)$ & $(0,0166)$ & $(0,0003)$ & $(0,0003)$ & $(0,0015)$ & $(0,0015)$ & $(0,0014)$ & $(0,0014)$ \\
\hline \multirow{2}{*}{ B1.2 } & $0,98 \mathrm{bA}$ & $0,99 \mathrm{bA}$ & $4,35 \mathrm{dA}$ & $4,09 \mathrm{eB}$ & $10,93 \mathrm{bA}$ & $11,07 \mathrm{bA}$ & $18,26 \mathrm{abA}$ & $13,36 \mathrm{~dB}$ \\
\hline & $(0,0207)$ & $(0,0205)$ & $(0,0003)$ & $(0,0003)$ & $(0,0018)$ & $(0,0018)$ & $(0,0010)$ & $(0,0014)$ \\
\hline \multirow{2}{*}{ C1.1 } & $0,57 \mathrm{deA}$ & $0,59 \mathrm{eA}$ & $4,70 \mathrm{bA}$ & $4,19 \mathrm{cB}$ & $6,34 \mathrm{eB}$ & $6,57 \mathrm{eA}$ & $9,70 \mathrm{fA}$ & $9,86 \mathrm{fA}$ \\
\hline & $(0,035)$ & $(0,0346)$ & $(0,0003)$ & $(0,0003)$ & $(0,0032)$ & $(0,0031)$ & $(0,0020)$ & $(0,0019)$ \\
\hline \multirow{2}{*}{ C1.2 } & $0,69 \mathrm{dA}$ & $0,65 \mathrm{deA}$ & $4,26 \mathrm{fB}$ & $4,28 \mathrm{aA}$ & $7,15 \mathrm{deA}$ & $7,21 \mathrm{dA}$ & $11,30 \mathrm{eA}$ & $11,20 \mathrm{eA}$ \\
\hline & $(0,0314)$ & $(0,0314)$ & $(0,0003)$ & $(0,0003)$ & $(0,0029)$ & $(0,0028)$ & $(0,0017)$ & $(0,0017)$ \\
\hline \multirow{2}{*}{ D1.1 } & $0,83 \mathrm{cB}$ & $0,88 \mathrm{cA}$ & $4,71 \mathrm{abA}$ & $4,31 \mathrm{aB}$ & $9,25 \mathrm{cB}$ & $9,81 \mathrm{cA}$ & $15,16 \mathrm{cB}$ & $17,33 \mathrm{bA}$ \\
\hline & $(0,0231)$ & $(0,0231)$ & $(0,0003)$ & $(0,0003)$ & $(0,0022)$ & $(0,0021)$ & $(0,0012)$ & $(0,0011)$ \\
\hline \multirow{2}{*}{ D1.2 } & $0,79 \mathrm{cdB}$ & $0,92 \mathrm{bcA}$ & $4,42 \mathrm{cA}$ & $4,23 \mathrm{bB}$ & $8,73 \mathrm{cB}$ & $10,21 \mathrm{bcA}$ & $17,06 \mathrm{bA}$ & $16,90 \mathrm{bA}$ \\
\hline & $(0,0258)$ & $(0,0222)$ & $(0,0003)$ & $(0,0003)$ & $(0,0023)$ & $(0,0020)$ & $(0,0011)$ & $(0,0011)$ \\
\hline \multirow{2}{*}{ E1.1 } & $0,59 \mathrm{deA}$ & $0,60 \mathrm{eA}$ & $4,75 \mathrm{aA}$ & $4,21 \mathrm{bcB}$ & 6,62 deA & $6,71 \mathrm{eA}$ & $15,16 \mathrm{cA}$ & $15,03 \mathrm{cA}$ \\
\hline & $(0,0342)$ & $(0,0338)$ & $(0,0003)$ & $(0,0003)$ & $(0,0031)$ & $(0,0030)$ & $(0,0012)$ & $(0,0013)$ \\
\hline \multirow{2}{*}{ E1.2 } & $0,64 \mathrm{deA}$ & $0,64 \mathrm{deA}$ & $4,27 \mathrm{fA}$ & $4,13 \mathrm{~dB}$ & $7,12 \mathrm{deA}$ & $7,16 \mathrm{eA}$ & $13,86 \mathrm{cdA}$ & $14,56 \mathrm{dA}$ \\
\hline & $(0,0316)$ & $(0,0317)$ & $(0,0003)$ & $(0,0003)$ & $(0,0029)$ & $(0,0028)$ & $(0,0014)$ & $(0,0013)$ \\
\hline
\end{tabular}

* Modelo Linear Generalizado proposto por Nelder e Weddeburn (1972);

** Modelo Linear Clássico sob distribuição Normal.

*** As médias seguidas da mesma letra minúsculas nas colunas não diferem entre si ao nível de 5\% de significância pelo teste Tukey;

ma letra maiúsculas nas linhas não diferem entre si ao nível de $5 \%$ de significância pelo teste Tukey.

Em relação ao SST foi possível verificar interferência da temperatura nos iogurtes A 1.1, B 1.2 e D 1.1, no entanto não foi possível estabelecer uma relação de proporcionalidade entre o SST e a temperatura (embora nos iogurtes A 1.1 e B 1.2 o SST diminuiu com o aumento da temperatura, no caso do D 1.1 houve o aumento do SST com a aumento da temperatura). Verificou-se influência do sabor dos iogurtes nos valores do SST nos seguintes grupos: A à $\left( \pm 27^{\circ} \mathrm{C}\right), \mathrm{B}$ à $\left( \pm 9^{\circ} \mathrm{C}\right), \mathrm{C}$ à $\left( \pm 9^{\circ} \mathrm{C}\right), \mathrm{D}$ à $\left( \pm 9^{\circ} \mathrm{C}\right)$ e $\mathrm{E}$ à $\left( \pm 27^{\circ} \mathrm{C}\right)$. Os valores do SST variaram entre 9,70 e 20,20 (Tabela 1).

\section{DISCUSSÃO}

Ao entrar em contato com o meio bucal substâncias ácidas promovem alteração no coeficiente de solubilidade do esmalte dentário, ocasionando perda de minerais nos elementos dentários em virtude do $\mathrm{pH}$ bucal não variar entre 5 e 5,5 (valor crítico para descalcificação dos cristais de hidroxiapatia $)^{10}$. O fato das bebidas ácidas serem deglutidas após ingestão e estarem em contato com a saliva, não exime o elemento dentário de sofrer desmineralização ${ }^{11}$. Para Silva et al. ${ }^{12} \mathrm{o}$ acometimento de erosão dental é determinada pela frequência da ingestão de bebidas ácidas, de modo que quanto maior o consumo, maiores os picos de desmineralização.

As glândulas salivares são responsáveis pela produção de saliva, substância que sofre influência do meio bucal, repercutindo no processo de descalcificação da estrutura dentária. A desmineralização é resultante do desequilíbrio nas concentrações de íons de cálcio e fosfato presente no fluxo salivar, ocasionada diretamente (quando bebidas ácidas banham a cavidade oral) ou indiretamente (pela ingestão de carboidratos). Os ácidos são capazes de ocasionar uma diminuição no $\mathrm{pH}$ bucal, de modo que a perda de minerais do esmalte dental resulta de um processo que visa promover a estabilização das concentrações dos íons de cálcio e fosfato ligadas a proteínas salivares ou presente na forma de complexos ${ }^{13}$.
Através do efeito tampão a saliva busca manter o pH bucal neutro (o que favorece a estabilização das concentrações de minerais da hidroxiapatita) ${ }^{14}$. Limpeza das estruturas dentais também consiste em funções desempenhadas pela saliva, contribuindo para a redução dos efeitos causados pelas bebidas ácidas ${ }^{15}$. Venables et al. ${ }^{16}$ citam que a redução do fluxo salivar compromete a limpeza e proteção dos elementos dentários, aumentando assim o risco de erosão.

Alimentos e bebidas que contêm leite e, por conseguinte, níveis elevados de cálcio e fosfato (por exemplo, leite com chocolate, iogurtes) têm mais probabilidade de ter um efeito de remineralização no esmalte dental. No estudo de Jensdottir et al. ${ }^{17}$ o consumo de balas ácidas com cálcio resultou em menor $\mathrm{pH}$ crítico e consideravelmente menor potencial erosivo do que os doces ácidos sem cálcio.

Ao entrar em contato com a cavidade oral, o flúor se liga aos cristais de hidroxiapatita, e quando em quantidade suficiente, torna $\mathrm{o}$ esmalte dentário resistente a desmineralização à um pH próximo de 4,5. A escovação com creme dental é considerada uma importante medida para minimizar os efeitos da erosão dental, no entanto, algumas precauções devem ser evidenciadas. A escovação deve ser evitada imediatamente após a ingestão de bebidas ácidas, em virtude da abrasividade favorecer a perda do esmalte dentário que encontra-se fragilizado ${ }^{18}$.

$\mathrm{Na}$ literatura estudos revelam a interferência da temperatura no processo de erosão dental ${ }^{10,19}$. Baseados na hipótese de que a temperatura influência na dissolução dos cristais de hidroxiapatita (em virtude de interferir na velocidade de difusão dos elementos químicos através do esmalte), Cavalcanti el al. ${ }^{20}$ (2010) mensurou o pH, a ATT e o SST de bebidas isotônicas expostas à $\left( \pm 9^{\circ} \mathrm{C}\right)$ e $\left( \pm 23^{\circ} \mathrm{C}\right)$, verificando o efeito modulador da temperatura em relação as variáveis em questão. No presente estudo a interferência da temperatura foi observada em relação ao $\mathrm{pH}$, a ATT, o AL e o SST. O período em que parte das bebidas foram expostas à temperatura ambiente ( 3 horas) foi escolhida levando em consideração o tempo em que os iogurtes seriam retirados da geladeira e consumidos pelas crianças no horário do intervalo escolar.

Em virtude de a concentração de hidrogênio ser inversamente proporcional a concentração de hidroxila, o pH causa interferência na dissolução da hidroxiapatita. $\mathrm{O}$ fato das concentrações de cálcio e fosfato serem dependentes do pH também é exposto como uma das evidências que estabelece a relação entre o processo de solubilidade e a erosão dental. Cálculos simples revelam que uma queda do pH de uma unidade dentro da faixa de sete a quatro dá origem a um aumento de sete vezes na solubilidade da hidroxiapatita $^{21}$.

Embora tenha sido encontrado valores distintos para o $\mathrm{pH}$, os dados do presente estudo corroboram com pesquisas científicas que analisaram a variável em questão ${ }^{2,9,22}$, onde todos os iogurtes apresentaram $\mathrm{pH}$ inferior ao valor crítico para dissolução de esmalte dentário (5- 5,5). Apesar do baixo $\mathrm{pH}$, a literatura mostra resultados conflitantes quanto ao efeito erosivo dos iogurtes. Enquanto alguns autores afirmam que os iogurtes não causam efeito erosivo ${ }^{23}$, um relatório mostrou que tais bebidas aumentam significativamente o desgaste e reduz a dureza do esmalte dental $^{24}$.

Para Farias et al. ${ }^{25}$ as indústrias de processamento contribuem para a diminuição dos índices de erosão dental 
ao reduzirem a quantidade de conservantes e substâncias acidulantes adicionadas às bebidas industrializadas. Quanto maior a concentração de substâncias acidulantes, maior a probabilidade do elemento dentário sofrer dissolução mineral.

A interferência dos sólidos solúveis totais no desenvolvimento da erosão dental é baseada no grau de viscosidade das bebidas, de modo que quanto maior o escoamento do líquido maior a aderência de componentes da dieta às estruturas dentais. Apesar de apresentar algumas desvantagens (perda de sensibilidade, dependência da temperatura), a refratometria vem sendo utilizada na comunidade científica para mensurar os sólidos solúveis totais de diversas bebidas ${ }^{2,22,26}$.

Embora o estudo explane através dos resultados a influência das propriedades físico-químicas (pH, ATT, AL e SST) no desenvolvimento da erosão dentária, os dados são apenas sugestivos, necessitando que novos estudos sejam realizados para ampliar a veracidade da pesquisa. Realizar testes adicionais in situ consiste em perspectivas de futuros estudos, a fim de demonstrar o comportamento do elemento dentário mediante exposição a iogurtes e sob efeito do fluxo salivar.

\section{CONCLUSÃO}

- Todos os iogurtes apresentaram $\mathrm{pH}$ inferior ao valor crítico para a dissolução do esmalte dentário $(5,5)$.

- Houve variação estatística significativa nos valores do pH, da acidez total titulável, do ácido lático e dos sólidos solúveis totais, quando iogurtes foram submetidos a diferentes temperaturas.

- Foram verificados nos iogurtes de sabores distintos diferenças estatísticas significativas nos valores das variáveis $\mathrm{pH}$, ATT, AL e SST.

- Os iogurtes mantidos refrigerados apresentaram menor acidez, motivo pelo qual sugeri- se que tais bebidas sejam consumidas geladas.

\section{REFERÊNCIAS}

1. Mangueira DFB, Passos IA, de Oliveira AFB, Sampaio FC. Erosão dentária: etiologia, diagnóstico, prevalência e medidas preventivas. Arq Odontol. 2016; 45(4):220-5.

2. Cavalcanti AL, Oliveira KFD, Paiva PS, Dias MVR, Costa SKP D, Vieira FF. Determinação dos sólidos solúveis totais ( ${ }^{\circ}$ Brix) e $\mathrm{pH}$ em bebidas lácteas e sucos de frutas industrializados. Pesqui Bras Odontopediatria Clín Integr. 2006; 6(1):57-64.

3. Prati C, Montebugnoli L, Suppa P, Valdrè G, Mongiorgi R. Permeability and morphology of dentin after erosion induced by acidic drinks. J Periodontol. 2003; 74(4):428-36.

4. Magalhães AC, Wiegand A, Rios D, Honório HM, Buzalaf MAR. Insights into preventive measures for dental erosion. J Appl Oral Sci. 2009; 17(2):75-86.

5. Sobral MAP, Adriana GT, Netto NG. Influence of the liquid acid diet on the development of dental erosion. Pesqui Odontol Bras. 2000; 14(4):406-10.

6. Nunn JH, Gordon PH, Morris AJ, Walker A. Dental erosion-changing prevalence? A review of British National childrens' surveys. International. J Paediatr Dent. 2003; 13(2):98-105.

7. Lussi A, Schlüter N, Rakhmatullina E, Ganss C. Dental erosion-an overview with emphasis on chemical and histopathological aspects. Caries Res. 2011; 45(Suppl 1):2-12.
8. Sivieri K, Oliveira MN. Avaliação da vida-de-prateleira de bebidas lácteas preparadas com "fat replacers" (Litesse e Dairy-lo). Rev Ciênc Tecnol Aliment. 2002; 22(1):24-31.

9. Zaze ACSF, Alves AEP, Bortolotti LV, Tondatti CA. Avaliação dos líquidos mais frequentimente encontrados na dieta de criança e análise de pH. Arq Ciências Saúde UNIPAR. 2011; 15(3):257-61.

10. Corso AC, Hugo FN, Padilha DMP. pH e titrabilidade ácida de sucos artificiais de limão. Rev Fac Odontol Porto Alegre. 2002; 43(1):30-3.

11. O'Sullivan EA, Curzon MEJ. Salivary factors affecting dental erosion in children. Caries Res. 2000; 34(1):82-7.

12. Silva JYBD, Brancher JA, Duda JG, Losso EM. Mudanças do $\mathrm{pH}$ salivar em crianças após a ingestão de suco de frutas industrializado. RSBO. 2008; 5(2):7-11.

13. Larsen MJ, Nyvad B. Enamel erosion by some soft drinks and orange juices relative to their $\mathrm{pH}$, buffering effect and contents of calcium phosphate. Caries Res. 1998; 33(1):81-7.

14. Jensdottir T, Nauntofte B, Buchwald C, Bardow A. Effects of sucking acidic candy on whole-mouth saliva composition. Caries Res. 2005; 39(6):468-74.

15. Jager DHJ, Vieira AM, Ligtenberg AJM, Bronkhorst E, Huysmans MCDNJM, Vissink A. Effect of salivary factors on the susceptibility of hydroxyapatite to early erosion. Caries Res. 2011; 45(6):532-7.

16. Venables MC, Shaw LINDA, Jeukendrup AE, RoedigPenman A, Finke M, Newcombe RG, Smith AJ. Erosive effect of a new sports drink on dental enamel during exercise. Med Sci Sports Exerc. 2005; 37(1):39-44.

17. Jensdottir T, Nauntofte B, Buchwald C, Bardow A. Effects of calcium on the erosive potential of acidic candies in saliva. Caries Res. 2006; 41(1):68-73.

18. Eisenburger M, Shellis RP, Addy M. Comparative study of wear of enamel induced by alternating and simultaneous combinations of abrasion and erosion in vitro. Caries Res. 2003; 37(6):450-5.

19. Barbour ME, Finke M, Parker DM, Hughes JA, Allen GC, Addy M. The relationship between enamel softening and erosion caused by soft drinks at a range of temperatures. J. Dent. 2006; 34(3):207-13.

20. Cavalcanti AL, Xavier AFC, Souto RQ, Oliveira MDC, Santos JAD, Vieira FF. Avaliação in vitro do potencial erosivo de bebidas isotônicas. Rev Bras Med Esporte. 2010; 16(6):455-8.

21. Smith AJ, Shaw L. Baby fruit juices and tooth erosion. Br Dent J. 1987; 162(2):65-7.

22. Lima HMR, Lima LR, Galvão FFDSP. Consumo infantil de bebidas lácteas: sólidos solúveis totais (Brix) e pH. Odontol Clín-Cient. 2011; 10(3):237-41.

23. Lussi A, Kohler N, Zero D, Schaffner M, Megert B. A comparison of the erosive potential of different beverages in primary and permanent teeth using an in vitro model. Eur J Oral Sci. 2000;108(2):110-4.

24. Jitpukdeebodintra S, Chuenarrom C, Muttarak C, Khonsuphap P, Prasattakarn S. Effects of $1.23 \%$ acidulated phosphate fluoride gel and drinkable yogurt on human enamel erosion, in vitro. Quintessence Int. 2010; 41(7):595-604.

25. Farias MMAG, Tames DR., Ferreira R, Bahi FC, Morreto J. Propriedades erosivas de sucos de frutas industrializados recomendados como suplemento alimentar para crianças. J Bras Odontopediatr Odontol Bebê. 2000; 3(12):111-7. 
26. Nogueira LC, Silva F, Ferreira IM, Trugo LC. Separation and quantification of beer carbohydrates by high-performance liquid chromatography with evaporative light scattering detection. J Chromatogr. 2005; 1065(2):207-10.

\section{CONFLITO DE INTERESSES}

Os autores declaram não haver conflitos de interesse.

\section{AUTOR PARA CORRESPONDÊNCIA}

Maria Helena Chaves de Vasconcelos Catão mhelenact@zipmail.com.br

Submetido em 08/03/2017 Aceito em 03/04/2017 